\title{
Structure and kinematics of galaxy clusters. I. The redshift catalogue ${ }^{\star}$
}

\author{
P. Stein ${ }^{\star \star}$ \\ Astronomisches Institut der Universität Basel, Venusstrasse 7, CH-4102 Binningen, Switzerland
}

Received July 19; accepted September 18, 1995

\begin{abstract}
An extensive redshift survey has been conducted on a sample of 15 nearby $(0.01 \lesssim z \lesssim 0.05)$ clusters of galaxies. A total number of 860 redshifts were determined by fitting of emission-lines and/or cross-correlation techniques. Of this sample, 735 galaxies are within $0.2-0.8 \mathrm{Mpc}\left(H_{0}=50 \mathrm{~km} \mathrm{~s}^{-1} \mathrm{Mpc}^{-1}\right)$ of the center of clusters. Approximate morphological types are available for most of the galaxies. A comparison of the present redshifts with published data allows an extensive error analysis. The agreement is excellent with the most modern data, showing a zero point error of $5 \mathrm{~km} \mathrm{~s}^{-1}$ and an overall consistency of the measurements and their uncertainties. We estimate our redshifts to have mean random errors around $30 \mathrm{~km} \mathrm{~s}^{-1}$. A population analysis of the clusters will be given in a forthcoming paper. ${ }^{\star \star \star}$
\end{abstract}

Key words: galaxies: clusters: general — galaxies: redshifts — catalogues

\section{Introduction}

During the last few years the number of known redshifts has undergone an exponential rise. This is due mainly to the strongly rising number of multi-fibre instruments in operation, and to the breakthrough of CCD detectors in combination with cross-correlation methods for measuring absorption-line redshifts.

Several redshift surveys aimed at studying galaxy clusters, which led to significant progress in the understanding of structural and dynamical aspects (e.g. Lucey et al. 1986; Dressler \& Shectman 1988; Beers etal. 1991b; Malumuth et al. 1992). Most of these studies concentrated on measuring redshifts for the brightest galaxies in regions extending $\gtrsim 1 \mathrm{Mpc}$ around the cluster centers. Thus little is known about the degree of substructure in the cores of galaxy clusters, an information which would greatly constrain theories of dark matter distribution and cluster formation and evolution (Merritt 1988). Other challenging questions about galaxy clusters remain yet unanswered. Among them: what is the kinematical status of galaxies of different types, especially as compared with the mor-

\footnotetext{
${ }^{\star}$ Based on observations made at the European Southern Observatory, La Silla, Chile

${ }^{\star \star}$ Present address: Departament d'Astronomia i Meteorologia, Universitat de Barcelona, Avenida Diagonal 647, E-08028 Barcelona, Spain. E-mail: paul at mizar.am.ub.es

${ }^{\star \star \star}$ Tables 1 and 2 are only available in electronic form at the CDS via anonymous ftp 130.79.128.5
}

phological segregation in projected space (Dressler 1980)? Which frequency and significance have peculiar velocities of $\mathrm{cD}$ galaxies? Is mass segregation in the very center of rich clusters a dominant effect?

As a contribution towards the solution of these problems we have measured redshifts for about $30-100$ galaxies in the central regions of several nearby galaxy clusters $(0.01 \lesssim z \lesssim 0.05)$. The nearness of our sample allows for raw, mainly new morphological types to be assigned using existing photographic material.

\section{Observations}

Selecting an unbiased and possibly complete sample of nearby galaxy clusters was difficult when this project began in 1988. The southern Abell cluster catalogue (Abell et al. 1989) was not yet published. Moreover, neither X-ray selected cluster catalogues (e.g. Gioia et al. 1990) nor catalogues based on automated selection criteria (e.g. Lumsden et al. 1992) were available at that time. Our cluster sample was chosen inspecting ESO survey plates by eye, and selecting the highest galaxy density peaks on them. Most of the clusters later turned out to fulfill Abell's criteria. This sample is representative in the sense that it contains a significant fraction of nearby clusters of different morphologies, richnesses and X-ray luminosities.

All galaxy spectra were obtained with the OPTOPUS multifiber instrument. The two observing runs on the $3.6 \mathrm{~m}$ telescope at ESO, La Silla, took place in August, 
1989 and April/May, 1990. The main characteristics of OPTOPUS are: a field of view of $33^{\prime}$ and as many as 30 (1989) respectively 50 (1990) fibers, each of $2.3^{\prime \prime}$ diameter. These fibers were plunged into holes that had been previously drilled into a solid plate. Two different sets of fibers were provided during the 1990 run, thus allowing to plunge one set of fibers into a drilled plate, while the other fiber set was performing at the telescope. This significantly reduced the time loss between two subsequent exposures. Excellent weather conditions allowed a total of 34 exposures in 8 nights to be taken. During these observations 1276 spectra were obtained in 20 fields. Although some of the fields revealed themselves as chance projections of partly unrelated galaxies in the plane of the sky, the great majority of the acquired data proved to be inside rich clusters of galaxies.

During the first observing run in 1989 a dispersion of $170 \AA \mathrm{mm}^{-1}$ (first order) was chosen, which together with a $1024 \times 640 \mathrm{CCD}$ and a pixel size of $15 \mu \mathrm{m}$ gives a nominal resolution of $2.55 \AA /$ pixel. The wavelength range was chosen between $3800 \AA$ and $5800 \AA$. In the 1990 run a dispersion of $224 \AA \mathrm{mm}^{-1}$ was preferred, which leads to a resolution of $3.36 \AA /$ pixel. A broader range in wavelength, between $3800 \AA$ and $6500 \AA$ was thus covered. On both occasions the inclusion of some bright emission lines $\left(\mathrm{H}_{\delta}\right.$, $\mathrm{H}_{\gamma}, \mathrm{H}_{\beta}$, O III) and most of the absorption features suitable for cross-correlation (Ca II H+K, G-band, $\mathrm{H}_{\beta}$, Mg I, in 1990 also $\mathrm{Na} \mathrm{D}$ and several iron lines) was guaranteed. For each field a single exposure with a duration of $70 \mathrm{~min}$ was taken. He-Ne wavelength calibrations as well as nightsky exposures preceded and followed each exposure.

\section{Data reduction}

A software package for automated processing of multifibre spectra and subsequent cross-correlation has been developed by the author for reducing the data. The only necessary on-line interactions are the measurement of emission line redshifts and also cutting evident emission lines prior to cross-correlation. The package consists mainly of FORTRAN programs with the addition of some MIDAS commands and runs inside the MIDAS environment. An important consequence of this automated processing is the homogeneity and reproducibility of the results. Using FORTRAN routines instead of MIDAS procedures for the main steps of the cross-correlation has the advantage of speeding up the reduction: on a $\mu \mathrm{VAX} 4000$ the time required to obtain redshifts from raw data is around 1 $\mathrm{min} /$ galaxy, once the setup is done.

Starting from raw CCD spectra several reduction steps were necessary, including cleaning from defects, onedimensional extraction, flat-fielding, wavelength calibration and sky subtraction. Thereafter, the spectral data were ready for redshift measurements, which was done either using emission lines or absorption lines or both, where available. A detailed description follows: a) After pre-reduction of the CCD image much care was spent on optimal extraction of the spectra. The algorithm used has been developed by Horne (1986), and is efficient in optimizing $S / N$ as well as in cosmic rays removal. The resulting one-dimensional spectra were

b) flat-fielded

and

c) transformed to a wavelength scale using $\mathrm{He}-\mathrm{Ne}$ lamp calibrations before and after each exposure, to correct for deflections in the spectrograph. Given the importance of a good wavelength calibration for the final result, several quality checks were applied, including control of the position of night-sky lines in galaxy spectra and selfconsistency in the position of different emission lines where more than one was found. A precision of better than $\pm 1 \AA$ everywhere in the spectrum was obtained, which translates to $70 \mathrm{~km} \mathrm{~s}^{-1}$ uncertainty per line. Afterwards, the spectra were

d) corrected for the characteristic transmission of the fibers and then

e) the sky was subtracted. Unfortunately, our night sky exposures were severely read-out noise limited. A better approximation for the sky contribution was provided by the faintest galaxy spectrum out of all those taken with the same OPTOPUS-exposure. The results were also tested with the second faintest galaxy and proved to be reliable for spectra with a $S / N$ above 7 . This was fulfilled in $70 \%$ of the cases. The reason for this high success rate is that the range in brightness between the faintest and the brightest galaxies in the small field of view is necessarily large and thus the faintest galaxies are strongly underexposed.

After these stages the spectra are finally ready for redshift measurement, either through fitting of known emission lines, or through cross-correlation against a template galaxy of known redshift, or both.

\subsection{Emission-line redshifts}

As a next step every spectrum was inspected for the presence of strong emission lines. If more than one was found, the redshifted position of each of them was determined through an interactive fitting procedure. Thereby a Gaussian superimposed onto a linear or quadratic polynomial, approximating the local continuum, was used. As expected, the most common emission lines found were $\mathrm{H}_{\beta}$ and the two O III lines (4959 and $5007 \AA$ ); in some cases also $\mathrm{O}$ II, Ne III, $\mathrm{H}_{\gamma}$ and $\mathrm{H}_{\delta}$. The largest errors of the emission line redshifts are due to the uncertainties in the fit as well as to bigger-than-average deviations in the wavelength calibration quality at the position of a given emission line. An error analysis of a subsample of emission line spectra shows that the measurement errors amount on average to about $100 \mathrm{~km} \mathrm{~s}^{-1}$ for each single line. As a consequence, an uncertainty of $100 / \sqrt{n} \mathrm{~km} \mathrm{~s}^{-1}$ was chosen for emission line redshifts, where $n$ stands for the number 
of emission lines per galaxy involved. A minimum of $n=2$ emission lines was required to avoid misidentifications.

\subsection{Cross-correlation redshifts}

With the exception of Seyferts and other galaxies with central activity, no emission lines are expected in the central region of a galaxy covered by the OPTOPUS fibers. Most spectra are bulge-dominated and characterized by strong absorption lines, upon which the cross-correlation method relies. This method was described in detail by e.g. Tonry \& Davis (1979). In recent years cross-correlation has become a standard procedure, mainly because of its precision and objective error analysis. The achieved accuracy will be discussed in Sect. 5. Prior to cross-correlation it is necessary to cut emission lines away and transform the spectral continuum to a constant level of zero. Special care was taken while using a high-pass filter in order to make this continuum subtraction. Residual features of low spatial frequency would result in being treated as broad, superposed "absorption lines", distorting the peak of the cross-correlation-function.

A fundamental problem using the cross-correlationmethod is the choice of one or more templates, because this defines directly the zero-point shift. There are divergent opinions on which kind of and on how many different templates to use (Teague et al. 1990; Malumuth et al. 1992; Bardelli et al. 1994). For the sake of speed and simplicity only one template was used here, which was constructed merging 20 galaxy spectra with high $S / N$. They were obtained during the same observing runs as the data to be reduced. In this way, systematic deviations due to instrument configuration and setup could be minimized. Moreover, we concluded from several tests that a template with careful zero-point determination, reasonable line widths, and a scaling of all larger absorption lines to the same depth is more decisive for the quality of the results than using several different stars and galaxies as templates.

Some of the reduction steps after spectra preparation are illustrated in Fig. 1, where a high $S / N$ spectrum is shown, as well as one that barely reached the level for inclusion into the redshift catalogue. A threshold of 0.25 was chosen for the height of a normalized cross-correlationpeak in order to flag the result as confident. This limit is meant to be conservative and was determined by examining the level below which cross-correlation-results became randomly distributed instead of lying in a realistic redshift range.

\section{Catalogue}

In cases where both emission and absorption line redshifts could be measured for the same galaxy, they always agreed within statistics and a weighted mean was taken.

The result of the different reduction steps and crosscorrelation described above is a catalogue with 860 red-
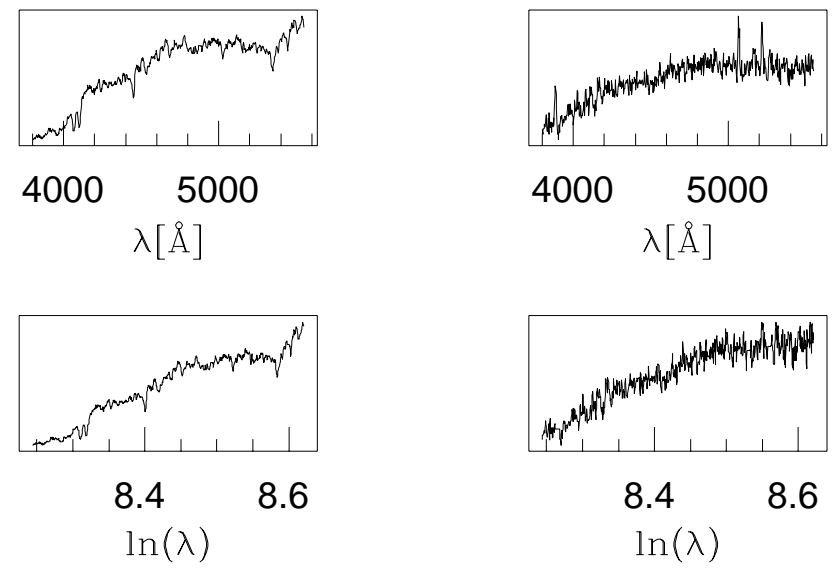

8.4

8.6
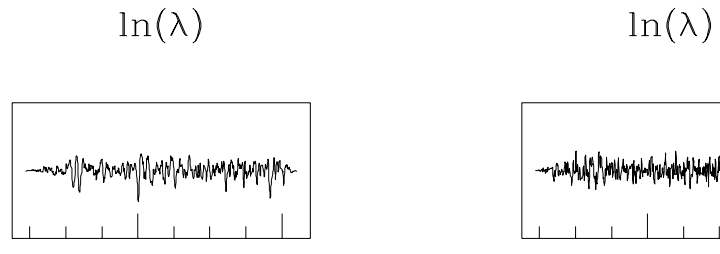

$8.4 \quad 8.6$

$\ln (\lambda)$

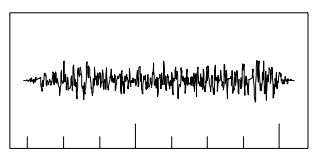

8.4

8.6

$\ln (\lambda)$

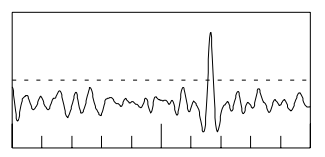
$-0.1$
0
0.1
$\ln (1+z)$

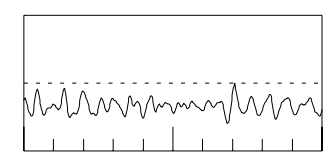
$-0.1$
0
$\ln (1+z)$

Fig. 1. Cross-correlation technique. Left an example for a spectrum with good $S / N$ and right a faint spectrum, which barely reaches the threshold for inclusion in the redshift catalogue. Strong emission lines are first eliminated from the pre-reduced spectra (top) and the spectra are rebinned logarithmically (2nd row). Then the continuum is subtracted (3rd row). These spectra are cross-correlated against a template and the resulting function plotted (bottom). If the maximum of the normalized cross-correlation-function fails to reach the dashed line with the value of 0.25 , the result is ignored

shifts. The data are listed in Tables 1 and 2 and velocity histograms are drawn for each cluster separately in Fig. 2. The galaxies have been divided into only three morphological classes, E, S0 and S, because of the impossibility to extract more information from direct plate examination. It should be stressed that this is not meant to be a definitive morphological classification. The correspondence between the author's classification and literature data, where available, is around $80 \%$. This information will be used in a forthcoming paper in order to separate statistically, using a large sample, kinematical effects into distinct morphological classes. 


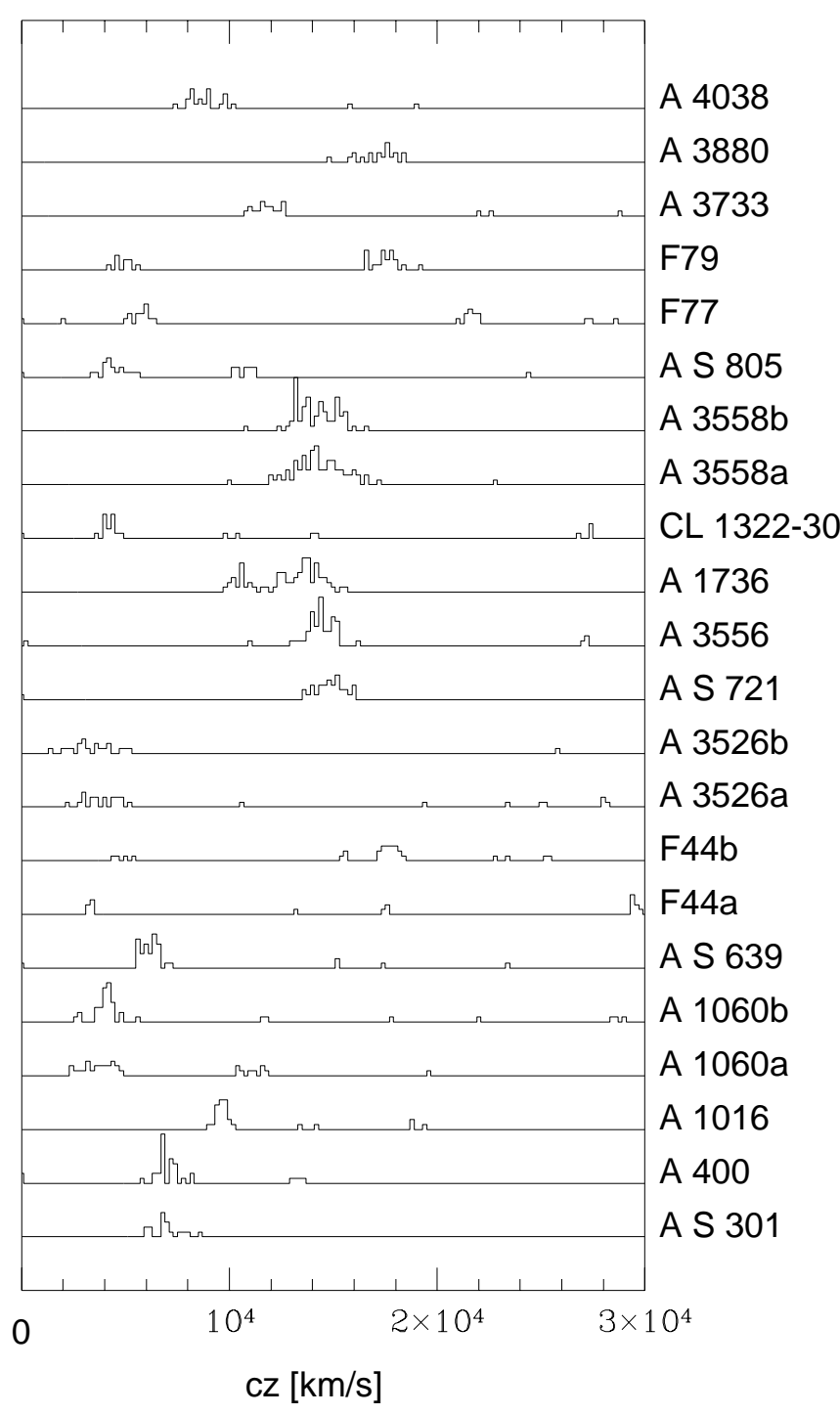

Fig. 2. Histogram of redshifts measured in this work, divided into OPTOPUS fields. In fields A S 301, A 400, A 3556, A 1736, A 3558a, A 3558b, A S 805, A 3733, A 3880 and A 4038 more than one exposure has been taken. The contribution of foreand background galaxies is clearly visible. The offset between zero-level lines in vertical direction amounts to 11 galaxies

\section{Redshift errors}

The measured redshifts can be affected by various error sources. For instance, poor flat-fielding could cause a spurious distortion of relevant lines and features. For faint sources good sky subtraction is of paramount importance. Moreover, the quality of the wavelength calibration depends directly on the spacing and on the $S / N$ of comparison lines. Some other minor error sources exist, like possible intrinsic dissimilarities between the spectra of template and object or inaccurate fitting of the cross-correlationpeak. All of these effects result in a distortion of the crosscorrelation-peak and are thus reflected in the estimation of redshift uncertainties (see Tonry \& Davis 1979 for a detailed description of error estimation).

With the cross-correlation-method only relative estimates for the random redshift errors are possible. It is therefore necessary to apply a scaling factor to these errors in order to obtain realistic values. In a first step such a scaling factor has been determined using those 36 galaxies which had been observed twice (see also Malumuth et al. 1992). Of course, the corrected errors, listed in Table 1 and 2, may still be smaller than the true external random errors. However, the subsequent comparison with redshifts from other sources in Sect. 6 and the resulting consistency can be taken as evidence that the errors listed in Tables 1 and 2 are close to the true external errors.

\section{Comparison of different redshift catalogues}

Given the nearness of our cluster sample, a considerable overlap with the literature was expected to arise. Indeed, the redshift overlap with external sources, which existed when this program was started, was much increased due to the publication of large amounts of additional data (Teague et al. 1990; Beers et al. 1991b; Malumuth et al. 1992). In addition, first results from running projects could be considered (Mazure 1994), which allowed the compilation of a comparison sample with more than 200 galaxies. These overlapping data are used in the following to estimate the external zero-point error and the true random errors of the galaxies in Table 1 as well as of those in external catalogues.

The procedure used for testing if two samples of redshift (radial velocity) measurements for the same galaxies were consistent within the errors goes as follows: let $v_{i}$ and $v_{j}$ be the radial velocities for the same galaxy from two sources; the quoted errors being $\Delta v_{i}$ and $\Delta v_{j}$, respectively. We define $\delta_{\mathrm{ud}}(i, j)$ as the pairwise unweighted differences between velocities, and $\delta_{\mathrm{wd}}(i, j)$ the same as $\delta_{\mathrm{ud}}(i, j)$, but weighted by the errors:

$$
\begin{gathered}
\delta_{\mathrm{ud}}(i, j)=v_{i}-v_{j} \\
\delta_{\mathrm{wd}}(i, j)=\frac{v_{i}-v_{j}}{\sqrt{\Delta v_{i}^{2}+\Delta v_{j}^{2}}}
\end{gathered}
$$

Instead of taking classical estimators like mean and standard deviation, measures of the central location $\mu$ and scale $\sigma$ for $\delta_{\text {ud }}$ and $\delta_{\mathrm{wd}}$ have been used which are less sensitive against outliers ("biweight estimators", see Beers et al. 1991a).

Central locations of $\delta_{\text {ud }}$ and $\delta_{\text {wd }}$ give a measure of the zeropoint shift between samples $i$ and $j$, while their spread is caused by the combined random errors $\Delta v_{i}$ and $\Delta v_{j}$. In case of negligible zero-point shift and of appropriate estimates of the random errors, $\delta_{\mathrm{wd}}(i, j)$ is expected to be Gaussian distributed around 0 with a standard deviation of 1 . The values $\delta_{\mathrm{wd}}$ can be interpreted as factors with 
Table 3. Internal and external comparison of redshifts. The comparison data was divided into three distinct groups: group a) included sources 2,3 , and 4 , the three most modern ones, presenting results for individual clusters. Group b) was built by sources 5,6 , and 7, which were somehow older but also dealing with specific clusters. In the third group (c) all sample catalogues were put together, mainly the RC3 (de Vaucouleurs et al. 1991) and several other sources from ZCAT (Huchra 1991). Column 3 gives the number of objects involved. Robust measures for central location and scale (Beers et al. 1991a) of unweighted differences are listed in Cols. 4 and 5, whereas the same parameters, but weighted by the redshift errors are listed in Cols. 6 and 7 . Values in Cols. 4 and 6 are positive if the redshift from the present work is bigger than the comparison redshift. The quoted intervals correspond to $68 \%$ confidence levels and were computed using a bootstrapping technique

\begin{tabular}{|c|c|c|c|c|c|c|c|}
\hline \multirow{2}{*}{$\begin{array}{l}\text { Seq. } \\
\text { (1) }\end{array}$} & \multirow{2}{*}{$\begin{array}{l}\text { Comparison } \\
\text { source } \\
(2)\end{array}$} & \multirow[b]{2}{*}{$\begin{array}{c}N_{\text {tot }} \\
(3)\end{array}$} & \multicolumn{2}{|c|}{ unweighted } & \multicolumn{2}{|c|}{ weighted } & \multirow{2}{*}{$\begin{array}{c}\text { group } \\
(8)\end{array}$} \\
\hline & & & $\begin{array}{c}\mu_{\mathrm{ud}} \\
(4)\end{array}$ & $\begin{array}{c}\sigma_{\mathrm{ud}} \\
(5)\end{array}$ & $\begin{array}{c}\mu_{\mathrm{wd}} \\
(6)\end{array}$ & $\begin{array}{c}\sigma_{\mathrm{wd}} \\
(7)\end{array}$ & \\
\hline 1 & cross-corr. vs. emission & 29 & $21 \pm 13$ & $67 \pm 9$ & $0.31 \pm 0.19$ & $0.99 \pm 0.16$ & \\
\hline 2 & Mazure (1994) & 31 & $-1 \pm 13$ & $65 \pm 8$ & $0.02 \pm 0.19$ & $0.96 \pm 0.11$ & \\
\hline 3 & Malumuth et al. (1992) & 20 & $5 \pm 14$ & $60 \pm 9$ & $0.02 \pm 0.26$ & $1.04 \pm 0.14$ & $\mathrm{a}$ \\
\hline 4 & Beers et al. (1991b) & 26 & $15 \pm 13$ & $67 \pm 10$ & $0.22 \pm 0.22$ & $1.09 \pm 0.20$ & \\
\hline 5 & Teague et al. (1990) & 43 & $5 \pm 16$ & $117 \pm 22$ & $0.02 \pm 0.22$ & $1.47 \pm 0.20$ & \\
\hline 6 & Dressler \& Shectman (1988) & 40 & $-45 \pm 12$ & $81 \pm 11$ & $-0.96 \pm 0.25$ & $1.70 \pm 0.24$ & $\mathrm{~b}$ \\
\hline 7 & Lucey et al. (1986) & 33 & $43 \pm 25$ & $140 \pm 19$ & $0.44 \pm 0.31$ & $1.84 \pm 0.35$ & \\
\hline 8 & $\mathrm{RC} 3$ & 52 & $-5 \pm 13$ & $101 \pm 13$ & $-0.31 \pm 0.34$ & $2.37 \pm 0.29$ & \\
\hline 9 & AAT \& Stromlo & 43 & $-4 \pm 10$ & $76 \pm 18$ & $0.04 \pm 0.30$ & $2.02 \pm 0.29$ & \\
\hline 10 & Fairall & 13 & $-27 \pm 32$ & $93 \pm 86$ & $-0.09 \pm 0.54$ & $1.84 \pm 0.33$ & $\mathrm{c}$ \\
\hline 11 & Las Campanas & 35 & $-38 \pm 15$ & $86 \pm 14$ & $-0.68 \pm 0.30$ & $1.69 \pm 0.25$ & \\
\hline 12 & Palumbo & 11 & $-127 \pm 101$ & $245 \pm 54$ & $-0.67 \pm 0.49$ & $1.31 \pm 0.23$ & \\
\hline 13 & $\mathrm{ESO}$ & 36 & $-35 \pm 32$ & $153 \pm 29$ & $-0.72 \pm 0.61$ & $3.40 \pm 0.70$ & \\
\hline
\end{tabular}

which the published redshift errors should be multiplied if the random errors in Table 1 are correct.

\subsection{Internal comparison}

In a first step, cross-correlation-redshifts were compared with emission line redshifts for those 29 galaxies for which both could be determined. The adopted random errors of the emission-line redshifts were discussed in Sect. 3.1; they amount to $60 \mathrm{~km} \mathrm{~s}^{-1}$ on average. The error of the crosscorrelation-redshifts becomes then, inverting Eq. (2), $34 \mathrm{~km} \mathrm{~s}^{-1}$. The zero-point difference of the two sets is 21 $\pm 13 \mathrm{~km} \mathrm{~s}^{-1}$ (the emission-line redshifts being smaller); this is hardly significant.

\subsection{External comparison}

The results of the internal check, as well as an extensive comparison between our redshifts and literature data are presented in Table 3. The data from the redshift collection of J. Huchra at the Center of Astrophysics, Harvard (ZCAT) were adopted with the original source given there. All velocities without quoted errors were given an uncertainty value of $100 \mathrm{~km} \mathrm{~s}^{-1}$.

Extraction of mean zero-point shift in the redshift of the present work was done using only catalogues of group (a), as well as from (a) and (b) together. The mean weighted zero-point shift amounts to $-3.9 \pm 5.8 \mathrm{~km} \mathrm{~s}^{-1}$ using only (a), and to $-6.9 \pm 4.4 \mathrm{~km} \mathrm{~s}^{-1}$ using (a) and (b) together. This shift is small and definitely consistent with 0 . Therefore no zero-point correction has been applied to the redshifts.

It can be seen that an excellent agreement exists when considering only those recent studies which used the crosscorrelation-technique and which concentrated on a few clusters (i.e. Mazure 1994; Malumuth et al. 1992; Beers et al. 1991b). Although the mean errors quoted by these three sources lie about 1.5-2 times higher than those given in the present work no discrepancy is found (Fig. $3)$. The probability that $\delta_{\mathrm{wd}}$ in this case is gaussian distributed with $\mu=0$ and $\sigma=1$ can be determined by a $\chi^{2}$-test and resulted to be $96 \%$.

If we took larger redshift errors for our data a discrepancy would arise: with errors a factor 1.3 bigger the $\chi^{2}$-test would give only $38 \%$ probability, and a factor of 1.7 would be consistent with $\sigma=1$ only with $5 \%$ probability. We conclude therefore that the most probable mean external error in radial velocity of our catalogue is around $30 \mathrm{~km} \mathrm{~s}^{-1}$, and in fact smaller than $45 \mathrm{~km} \mathrm{~s}^{-1}$ with $95 \%$ confidence. An excellent consistency between several redshift catalogues is thus found at this level of accuracy (compare discussion in Malumuth et al. 1992). It should be stressed that this conclusion is supported by as many as three independent sources with a total of 77 objects involved. 

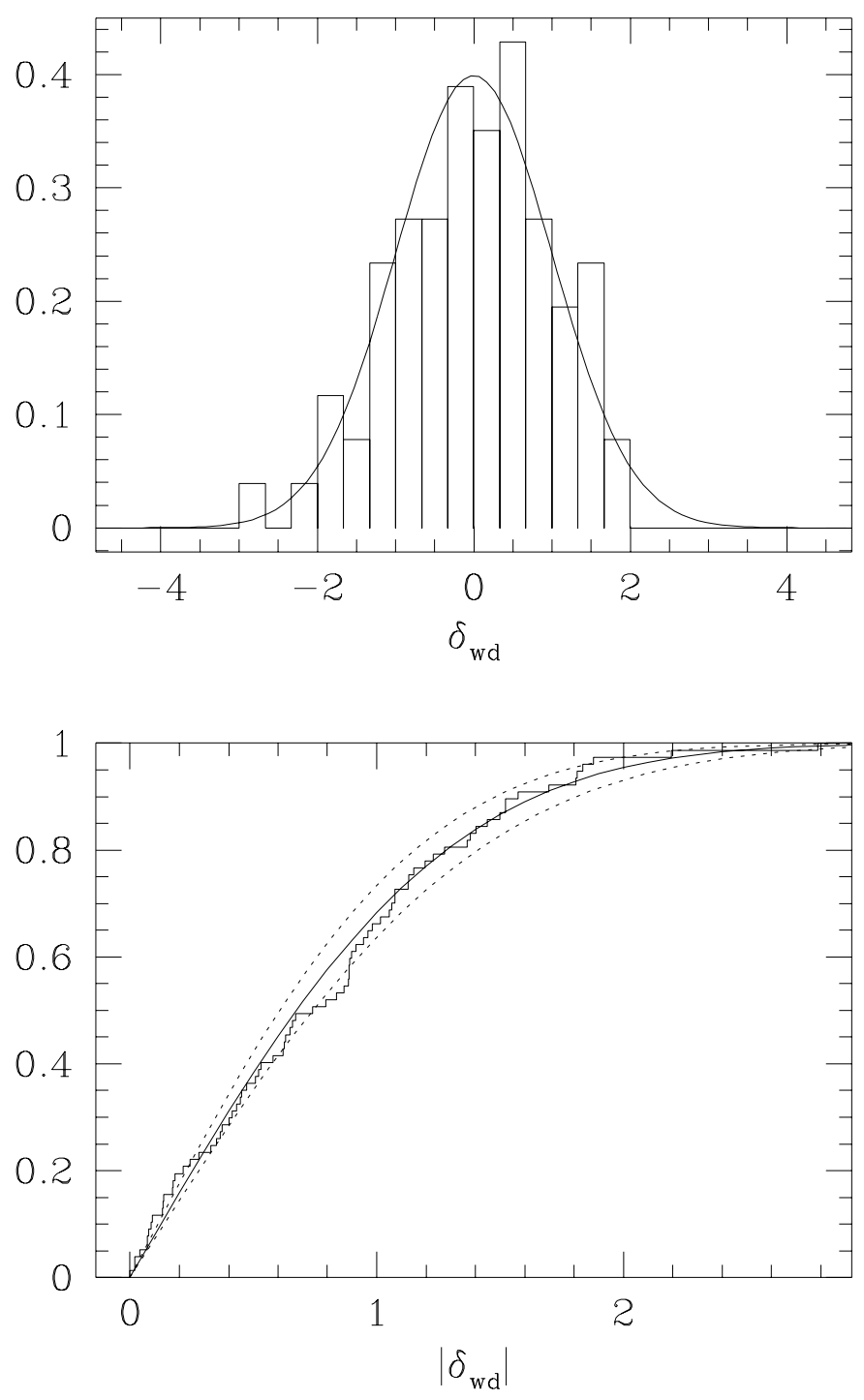

Fig. 3. Top: comparison between the redshift of the present work and those of Mazure (1994), Malumuth et al. (1992) and Beers et al. (1991b). The histogram represents the differences weighted by the errors quoted in Table 1 . The overlayed distribution is expected, under the assumption that all sources state realistic external redshift errors. Bottom: Kolmogoroff-Smirnov test. The probability that the histogram is drawn from the overlayed gaussian distribution is $66 \%$; a $\chi^{2}$-test gave $96 \%$ (see text). The dashed lines are cumulative gaussians with $\sigma=0.9$ resp. 1.1

Consequently, in view of the following considerations, we take our scaling factor for conversion to external errors as confirmed and interpret any residual discrepancy as arising from the accuracy of the external data. It can be seen in Table 3 that the only source revealing a significant zeropoint shift $\left(45 \mathrm{~km} \mathrm{~s}^{-1}\right)$ relative to our data is Dressler \& Shectman (1988). Low values for the absolute differences $\delta_{\mathrm{ud}}$ are found for Dressler \& Shectman (1988), AAT \& Stromlo and Las Campanas, meaning measurements of high relative precision. Their quoted uncertainties seem to be underestimations, though, as can be seen from the value of $\delta_{\mathrm{wd}}$. On the contrary, the catalogue of Palumbo et al. lists radial velocities with modest accuracy, but the quoted random errors seem to be realistic. Finally, Teague et al. (1990) as well as Fairall include data of very inhomogeneous quality in their catalogues. In general, there are some indications for those galaxies with the largest errors $\left(100-200 \mathrm{~km} \mathrm{~s}^{-1}\right)$ to be less accurate than stated, which could be explained by the fact that errors of this magnitude are mostly rough estimates.

\section{Conclusions}

New redshift measurements for 735 galaxies in the central regions $\left(R=0.2-0.8 \mathrm{Mpc}\right.$ for $\left.H_{0}=50 \mathrm{~km} \mathrm{~s}^{-1} \mathrm{Mpc}^{-1}\right)$ of 15 nearby clusters have been presented. Given a rather large overlap with existing data, and the homogeneity of the present measurements, it was shown that this redshift catalogue can be used for redshift errors calibration purposes, offering a link between numerous modern catalogues. A comparison of only the most recent and highestquality datasets shows that a mean external precision of $\lesssim 45 \mathrm{~km} \mathrm{~s}^{-1}$ has been achieved with a probability of $95 \%$. In addition, raw morphological types have been determined for most of the sample galaxies, using existing photographic material. These data will be used in a forthcoming paper in order to study substructures, as well as kimematics of galaxy clusters in dependence of luminosity and morphological types.

Acknowledgements. The author thanks Drs. A. Schröder and especially B. Leibundgut for their extensive contributions in the early stages of this project. I am grateful to Proff. I. Appenzeller and R. Bender for their hospitality at the Landessternwarte Heidelberg and for many stimulating discussions. Also, I wish to thank Prof. G.A. Tammann for his support throughout the duration of this project and for careful reading of the manuscript. Support of the Swiss National Science Foundation is gratefully aknowledged.

\section{References}

Abell G.O., Corwin H.G. Jr., Olowin R.P., 1989, ApJS 70, 1

Bardelli S., Zucca E., Vettolani G., et al. , 1994, MNRAS 267, 665

Beers T.C., Flynn K., Gebhardt K., 1991a, AJ 100, 32

Beers T.C., Forman W., Huchra J.P., Jones C., Gebhardt K., 1991b, AJ 102, 1581

de Vaucouleurs G., de Vaucouleurs A., Corwin H.G. Jr., et al., 1991, Third Reference Catalogue of Bright Galaxies. Springer Verlag, New York

Dressler A., 1980, ApJ 236, 351

Dressler A., Shectman S.A., 1988, AJ 95, 284

Gioia I.M., Maccacaro T., Schild R.E., et al., 1990, ApJS 72, 567

Horne K., 1986, PASP 98, 609 
Huchra J., 1991, ZCAT, computer readable version taken from DIRA Database, Bologna, 1993

Lucey J.R., Currie M.J. Dickens R.J., 1986, MNRAS 221, 453

Lumsden S.L., Nichol R.C., Collins C.A., Guzzo L., 1992, MNRAS 258, 1

Malumuth E.M., Kriss G.A., Van Dyke Dixon W., Ferguson H.C., Ritchie C., 1992, AJ 104, 495

Mazure A., 1994 (private communication)
Merritt D., 1988, The Minnesota Lectures on Clusters of Galaxies and Large Scale Structure, PASPC. In: Dickey J.M. (ed.)

Nilson P., 1973, Uppsala General Catalogue of Galaxies, Uppsala

Teague P.F., Carter D., Gray P.M., 1990, ApJS 72, 715

Tonry J., Davis M., 1979, AJ 84, 1511 